\title{
Probiotics in the prevention and treatment of atopic dermatitis
}

\author{
Sofia D'Elios $^{1}$ | Irene Trambusti ${ }^{1}$ | Elvira Verduci ${ }^{2}$ | Giuliana Ferrante ${ }^{3}$ | Sara Rosati ${ }^{1}$ | \\ Gian Luigi Marseglia ${ }^{4}$ (D) | Lorenzo Drago ${ }^{5}$ | Diego G. Peroni ${ }^{1}$ (I)
}

${ }^{1}$ Department of Clinical and Experimental Medicine, Section of Pediatrics, University of Pisa, Pisa, Italy

${ }^{2}$ Department of Health Science, University of Milan, Milan, Italy

${ }^{3}$ Department of Science for Health Promotion and Mother and Child Care, University of Palermo, Palermo, Italy

${ }^{4}$ Department of Pediatrics, Foundation IRCCS Policlinico San Matteo, University of Pavia, Pavia, Italy

${ }^{5}$ Clinical Microbiology, University of Milan, Milan, Italy

\section{Correspondence}

Peroni Diego, Department of Clinical and Experimental Medicine, Section of Paediatrics, Via Roma 67, 56126, Pisa, Italy. Email: diego.peroni@unipi.it

Editor: Gian Luigi Marseglia

\begin{abstract}
The use of probiotic supplements might change the composition of the intestinal flora of children, subsequently modulating the immune system's reactivity. The effects of probiotic administration for the prevention/treatment of allergic diseases and atopic dermatitis, in particular, are still so controversial that no definitive recommendation can be made at this stage. Differences in strain specificity, timing, and length of administration all contribute to diversifying the conclusions of this review.
\end{abstract}

\section{KEYWORDS}

allergic diseases, atopic dermatitis, child, eczema, infant, probiotics

\section{1 | INTRODUCTION}

The early microbiota is proposed to have a role in the pathogenesis of atopic dermatitis (AD). ${ }^{1}$ Therefore, it has been argued that modulating the host's gut microbiota could be an approach for preventing and/or treating allergic diseases. Among different factors that can modulate the gut microbiota to lead to preventive or therapeutic effects on pediatric allergic diseases, probiotic supplementation has been currently discussed with controversial results. Several reasons can be suggested for this, which may include the choice of the "right" probiotic, the dosage, the timing (window of opportunity), and the presence of many confounding factors that influence the success of a probiotic supplementation such as diet, age, obesity, ethnicity, and other environmental exposures.

\section{2 | METHODS}

The present review provides an overview of the recent evidence from the literature on the efficacy of the most common studied probiotic strains for the prevention and treatment of atopic dermatitis. Evidence published in the last five years has been searched using the MEDLINE and Cochrane Library; specific and overall strains, age, diseases, and type of publication have been used for the research. The search strategy on April 4, 2020, included: ((atopic dermatitis) OR eczema) AND probiotics. Filters: Full text, 5 years, Humans, English, Child: birth18 years. The systematic literature review identified 74 potentially relevant references. In total, 45 irrelevant papers were excluded after screening the titles. After reviewing the abstracts or full texts, nine studies were excluded. Thus, the actual review includes 20 articles. In 
this paper, we decided to analyze 14 of 20 articles, selecting the most recent and relevant. The data on $A D$ are presented according to the results of randomized, double-blind controlled trials (RDBCT), which analyzed the effects of mono- or multistrain probiotics, with a final discussion of reviews and meta-analyses. We have reported prevention studies first and then those on treatment.

\section{3 | RESULTS}

\section{1 | Monostrain probiotics}

Administration of the strain Bifidobacterium dentium in mothers from 36-week gestation to 3 months post-partum reduced the incidence of $A D$ in offspring by $40 \%$. The group responding to the probiotic intervention had a gut microbiota similar to the non-atopic children. ${ }^{2}$ In a RDBCT using Lactobacillus rhamnosus HN001 or Bifidobacterium lactis HN019 taken (dose 6X10 ${ }^{9}$ colony-forming units (CFU) daily) from 35 weeks of gestation to 6 months post-partum in mothers while breastfeeding and from birth to age 2 years in infants, the authors showed that HNO01 significantly protected against the development of eczema at 2, 4, 6, and 11 years and atopic sensitization at 6 years, while the strain HN019 had no significant effect on these outcomes. ${ }^{3}$ Administering Lactobacillus GG supplementation for the first 6 months of life (dose: $10^{10} \mathrm{CFU}$ daily), Cabana et al investigated the cumulative incidence of eczema in high-risk children (with at least 1 biologic parent who reported a history of asthma), ${ }^{4}$ but they were not able to demonstrate any preventive effect in the treated children. Lactobacillus rhamnosus (LR MP108) (dose $350 \mathrm{mg}$ daily) in children aged 4-48 months for 8 weeks reduced the clinical scores. However, the amount of topical corticosteroids showed no difference between groups, nor was there any significant difference noted in the overall symptom-free duration. ${ }^{5}$ Yan et al demonstrated that the probiotic $L$ paracasei $(1 \times 1010$ CFU daily) was not beneficial as a complementary approach to topical corticosteroids in infants with $A D$; however, slight beneficial effects may have been masked by the moderate potency of the corticosteroid used. ${ }^{6}$

\section{2 | Multistrain probiotics}

In a RDBCT from 36 weeks of gestation until 3 months post-partum, 415 pregnant women were randomized to receive either probiotics (LGG, $L$ acidophilus La-5, and $B$ animalis subsp lactis Bb-12 dose: $5 \times 10^{10} \mathrm{CFU}$ daily) or placebo. At 6 years, there was a trend toward a lower cumulative incidence of $A D$ in the probiotic group, but the prevalence of asthma and atopic sensitization was not significantly affected by the probiotic regime. ${ }^{7}$ In a RDBCT, a total of 290 participants were randomized to receive a daily mixture of LGG and B animalis subsp lactis BB-12 each at a dose of $10^{9} \mathrm{CFU}$, or placebo. During the follow-up (mean age: 16.1 months), a significantly lower incidence of eczema was observed in the probiotic group (4.2\%). ${ }^{8}$

\section{Key Messages}

This review showed that probiotic supplementation during the prenatal and postnatal periods seems to reduce the incidence of $A D$ in infants and children in both high-risk and unselected subjects, especially beginning in gestation through the first 6 months of life

In a 12-week RDBCT in children aged 4 to 17 years with moderate $A D$, a mixture of $B$ lactis CECT 8145, B longum CECT 7347, and $L$ casei CECT 9104 (dose: $10^{9}$ CFU daily) was able to produce a mean reduction in the SCORAD index and in the use of topical steroids. ${ }^{9}$ $L$ paracasei and $L$ fermentum either alone or combined (dose: $4 \times 10^{9}$ CFU daily), or placebo were given to 220 children for 3 months; the group who received the probiotic alone or combined showed lower SCORAD scores than the placebo group; this difference remained stable at four months after discontinuing the supplementation. ${ }^{10}$

\section{3 | Meta-analyses and reviews}

A meta-analysis from the World Allergy Organization demonstrated that probiotics used during the last trimester of pregnancy or in breastfeeding mothers taking probiotic supplementation could reduce the risk of eczema in infants, although the certainty of the evidence was low. This may be due to the risk of bias across trials, indirectness of the evidence, inconsistency of results among studies, and the imprecision of the pooled estimates. ${ }^{11}$

$\mathrm{Li}$ et al observed that the use of mixtures of probiotics including strains of Lactobacillus (OR: 0.64; 95\% Cl: 0.51-0.81), Bifidobacterium (OR: 0.63; 95\% Cl: 0.50-0.79), and Propionibacterium (OR: 0.80; $95 \% \mathrm{Cl}: 0.66-0.96)$ all appeared to reduce the incidence of $A D$ during both the prenatal and the postnatal period, from birth to 6 months of age (OR: $0.67 ; 95 \% \mathrm{Cl}$ : 0.54-0.82). ${ }^{12}$

Zuccotti et $\mathrm{al}^{13}$ showed that infants treated with a mixture of probiotics containing Lactobacilli and Bifidobacteria strains were effective in reducing the incidence of eczema in the first 24 months of life, with a partial loss of efficacy after 2 years of age, while no effect was documented for Lactobacilli and Bifidobacteria products alone.

Zhao et al analyzed eight clinical trials (741 infants) and revealed that the overall pooled change in the Scoring Atopic Dermatitis index $(95 \% \mathrm{Cl})$ in infants treated with Lactobacillus strains was -5.71 (-8.37, -3.04), $P<.01 .{ }^{14}$ Subgroup analysis revealed that the effect was $-8.32(-16.35,-0.28)$ in moderate-to-severe patients and higher for treatment duration $<8$ weeks $(-8.65(-14.32,-2.98))$.

A recent Cochrane Review ${ }^{15}$ included 39 randomized controlled trials with 2599 randomized participants of either gender, aged from the first year of life through to 55 years (only six studies assessed adults). ${ }^{1}$ The probiotics included were bacteria of the Lactobacillus and Bifidobacteria species taken alone or in combination with other probiotics for a period ranging from four weeks up to six months. 
Analysis of the total SCORAD scores by age-group showed no significant differences in total SCORAD between probiotic and control treatments among those under two years of age (MD: $-0.99 ; 95 \%$ $\mathrm{Cl}$ : -3.97 to 1.99 ; IR = 68\%); however, data from older age-groups showed a significant difference in favor of probiotics (age: 2 to 12 years; MD: -6.08 ; $95 \% \mathrm{Cl}:-9.68$ to -2.48 ; IR = 0\%). This evidence also suggests that probiotic supplementation may do little to improve the quality of life in some patients with eczema. Therefore, the use of probiotics needs to be further investigated with appropriate, well-designed trials.

\section{4 | DISCUSSION}

The effects of probiotic administration for the prevention/treatment of allergic diseases are still so controversial that no definitive recommendation can be made at this stage. Differences in strain specificity, timing, and length of administration all contribute to diversifying the conclusions of meta-analyses. The use of probiotic supplements might change the composition of the intestinal flora of children, subsequently modulating the immune system's reactivity and possibly playing an important role in AD prevention. This review showed that probiotic supplementation during the prenatal and postnatal periods seems to reduce the incidence of $A D$ in infants and children in both high-risk and unselected subjects, especially from gestation through the first 6 months of life. In the future, more rigorous, double-blind, and larger, well-designed RCTs are required to conclusively evaluate the efficacy of probiotics to prevent $A D$ and to explore the essential mechanisms. Researchers should also consider studying subgroups of patients, standardizing doses/concentrations, and the type of probiotic.

\section{ORCID}

Gian Luigi Marseglia (iD https://orcid.org/0000-0003-3662-0159

Diego G. Peroni (iD https://orcid.org/0000-0002-0234-1373

\section{REFERENCES}

1. Peroni DG, Nuzzi G, Trambusti I, Di Cicco ME, Comberiati P. Microbiome Composition and Its Impact on the Development of Allergic Diseases. Front Immunol. 2020 Apr 23; 11:700.

2. Avershina E, Cabrera Rubio R, Lundgård K, et al. Effect of probiotics in prevention of atopic dermatitis is dependent on the intrinsic microbiota at early infancy. J Allergy Clin Immunol. 2017;139: 1399-1402.e8.
3. Wickens K, Barthow C, Mitchell EA, et al. Effects of Lactobacillus rhamnosus HNOO1 in early life on the cumulative prevalence of allergic disease to 11 years. Pediatr Allergy Immunol. 2018;29:808-814.

4. Cabana MD, McKean M, Caughey AB, et al. Early probiotic supplementation for eczema and asthma prevention: a randomized controlled trial. Pediatrics. 2017;140:e20163000.

5. Wu YJ, Wu WF, Hung CW, et al. Evaluation of efficacy and safety of Lactobacillus rhamnosus in children aged 4-48 months with atopic dermatitis: An 8-week, double-blind, randomized, placebo-controlled study. J Microbiol Immunol Infect. 2017;50:684-692.

6. Yan DC, Hung $\mathrm{CH}$, Sy LB, et al. A randomized, double-blind, placebo-controlled trial assessing the oral administration of a heattreated Lactobacillus paracasei supplement in infants with atopic dermatitis receiving topical corticosteroid therapy. Skin Pharmacol Physiol. 2019;32:201-211.

7. Simpson MR, Dotterud CK, Storrø O, et al. Perinatal probiotic supplementation in the prevention of allergy related disease: 6 year follow up of a randomised controlled trial. BMC Dermatol. 2015;15:13.

8. Schmidt RM, Pilmann Laursen R, Bruun S, et al. Probiotics in late infancy reduce the incidence of eczema: a randomized controlled trial. Pediatr Allergy Immunol. 2019;30:335-340.

9. Navarro-López V, Ramírez-Boscá A, Ramón-Vidal D, et al. Effect of oral administration of a mixture of probiotic strains on SCORAD Index and use of topical steroids in young patients with moderate atopic dermatitis: a randomized clinical trial. JAMA Dermatol. 2018;154:37-43

10. Wang IJ, Wang JY. Children with atopic dermatitis show clinical improvement after Lactobacillus exposure. Clin Exp Allergy. 2015;45:779-787.

11. Cuello-Garcia CA, Brożek JL, Fiocchi A, et al. Probiotics for the prevention of allergy: A systematic review and meta-analysis of randomized controlled trials. J Allergy Clin Immunol. 2015;136:952-961.

12. Li L, Han Z, Niu X, et al. Probiotic supplementation for prevention of atopic dermatitis in infants and children: a systematic review and meta-analysis. Am J Clin Dermatol. 2019;20:367-377.

13. Zuccotti G, Meneghin F, Aceti A, et al. Probiotics for prevention of atopic diseases in infants: systematic review and meta-analysis. Allergy. 2015;70:1356-1371.

14. Zhao M, Shen C, Ma L. Treatment efficacy of probiotics on atopic dermatitis, zooming in on infants: a systematic review and meta-analysis. Int J Dermatol. 2018;57:635-641.

15. Makrgeorgou A, Leonardi-Bee J, Bath-Hextall FJ, et al. Probiotics for treating eczema. Cochrane Database Syst Rev. 2018;(11):CD006135.

How to cite this article: D'Elios S, Trambusti I, Verduci E, et al. Probiotics in the prevention and treatment of atopic dermatitis. Pediatr Allergy Immunol. 2020;31(Suppl. 26):4345. https://doi.org/10.1111/pai.13364 